\title{
Optical gain measurements based on fundamental properties and comparison with many-body theory
}

\author{
T. Keating, S. H. Park, ${ }^{\text {a) }}$ J. Minch, ${ }^{\text {b) }}$ X. Jin, and S. L. Chuang ${ }^{\text {() }}$ \\ Department of Electrical and Computer Engineering, University of Illinois at Urbana-Champaign, \\ 1406 West Green Street, Urbana, Illinois 61801 \\ T. Tanbun-Ek ${ }^{\text {d) }}$ \\ Bell Laboratories, Lucent Technologies, 700 Mountain Avenue, Murray Hill, New Jersey 07974
}

(Received 28 September 1998; accepted for publication 8 June 1999)

\begin{abstract}
We present high accuracy measurements of gain, loss, and transparency energy in long-wavelength semiconductors based on a hybrid approach using the fundamental relationship between the gain and the spontaneous emission spectra. Independent measurements of optical gain, transparency energy, and loss show the accuracy and validity of this technique. These results are compared with those obtained by the non-Markovian gain model with many-body effects under the spontaneous emission transformation method. It is found that the hybrid approach for the gain spectrum alleviates many of the problems related to the poor signal to noise ratio in the amplified-spontaneous emission near and below the band edge. The theoretical spectra compare well with the measured spectra for both the transverse electric and transverse magnetic polarizations. (C) 1999 American Institute of Physics. [S0021-8979(99)01018-X]
\end{abstract}

\section{INTRODUCTION}

Optical gain and loss are of fundamental importance to the study of lasers, since the gain determines many device performance characteristics. Therefore, accurate and reliable gain measurement techniques are essential tools for the experimental study of lasers. Likewise, highly accurate theoretical gain models incorporating the most realistic physical effects are also essential to advance the state of the art. Such models are most stringently tested against the best obtainable data, since the usefulness of theoretical predictions of gain and carrier density depends both on the model and on close agreement with accurately measured data.

On the experimental side, several excellent and widely used techniques already exist for measurements of gain and loss, yet there are instances where improvements are still needed. Measuring the gain in a Fabry-Perot or distributedfeedback laser typically first involves measuring the amplified spontaneous emission (ASE) of the laser. Since the ASE signals are very weak below the band edge, however, the evaluation of gain may degrade strongly due to poor signal to noise ratios in the ASE. As a consequence, commonly used techniques for measuring the modal gain, such as the Hakki-Paoli method ${ }^{1}$ or Cassidy's ${ }^{2}$ method, show a significant amount of noise in the region of the gain tail because of reduced signal intensity. While some reduction in the noise susceptibility of both methods can be achieved through an

\footnotetext{
${ }^{a}$ On leave from the Department of Physics, Catholic University of TaeguHyosang, Hayang, Kyeongbuk, Korea.

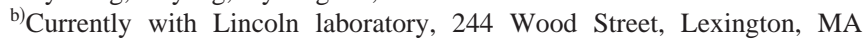
02420.

c) Author to whom correspondence should be addressed; electronic mail: s-chuang@uiuc.edu

d)Also with Multiplex, Inc. 115 Corporate Blvd., South Plainfield, NJ 07080.
}

averaging procedure, ${ }^{3}$ the results thus obtained are less accurate.

Difficulties in measuring the gain tail can compound to create further problems, since estimates of the intrinsic loss are often obtained by examining the limit of the gain tail. For instance, assuming the intrinsic losses are constant with respect to wavelength, the measured loss may be used to find the transparency energy. But it is impossible to do so if the losses are inaccurate. In turn each of these problems impacts the comparison between theory and experiment. Accurate values of the loss are needed to properly renormalize theoretical spectra. Moreover, an incorrect renormalization will, in effect, shift the location of the transparency energy. Thus, clear data in the tail region is necessary for comparison to theoretical gain models in terms of predicted line broadening behavior.

On the theoretical side, conventional optical gain calculations are usually based on the density-matrix theory with a phenomenological damping term which gives the Lorentzian line shape function. ${ }^{4}$ A few groups ${ }^{5-8}$ have argued that the optical gain spectra calculated with the Lorentzian line shape function deviate from the experimental results. Especially, an anomalous absorption region below the band gap and discrepancies between the transparency points and the Fermilevel separation appear in the gain spectra when the Lorentzian line shape is used. To overcome these artifacts, the intraband relaxation times by the carrier-carrier and carrierlongitudinal optical (LO) phonon scatterings have been considered to obtain the line shape function. ${ }^{7,8}$ Its low-energy tail decreases much faster than the Lorentzian line shape while its high-energy tail is close to the Lorentzian line shape. This shows that the steepness of the low-energy tail of the line shape function is most important. However, these models require a large amount of computational time. As a 
simplified approach, approximate formula considering only the hole-hole scattering has often been used in calculating the optical gain spectrum. ${ }^{9-11}$ Another approach is to replace the Lorentzian line shape function with a sharper spectral function, i.e., with a Gaussian line shape. The effect of Gaussian line shape is similar to that of the line shape function considering the carrier-carrier and carrier-LO phonon scattering.

On the other hand, the spontaneous emission transformation method using the fundamental relations between the spontaneous emission and the absorption coefficients has been proposed as an improved method. ${ }^{5,12-15}$ It guarantees that artifacts mentioned above are circumvented independent of the line shape functions (Lorentzian, Gaussian, or others). Also, the slow convergence of the Lorentzian leads to a very long tail of the gain spectra into the band gap, in contradiction to experimental observations. ${ }^{15}$ Hence, a non-Markovian (Gaussian) gain model under the spontaneous emission transformation method is desirable to obtain the optical gain spectrum. ${ }^{16,17}$

In this article, we present high accuracy measurements of the optical gain, using the fundamental relationship between the spontaneous emission spectrum and the gain spectrum. The values for transparency energy and loss obtained from this technique compare favorably with those obtained from several independent measurements. ${ }^{18}$ Experimentally, the major techniques involve measuring (1) gain and spontaneous emission, (2) polarization resolved gain spectra, (3) optical-pump induced modified gain spectra, and (4) pumpinduced electrical responses ${ }^{19}$ for directly obtaining the transparency energy and losses. The key information each technique provides is summarized as follows. First, since spontaneous emission and gain are related by only three parameters, the transparency energy, the loss, and a coupling constant, matching gain and spontaneous emission provides a way to estimate these parameters. Second, the quasi-Fermi level separation is the same at a fixed injection current for each polarization, so the polarization-resolved gain spectra will cross at the same quasi-Fermi level separation. Third, the net absorption of an optical pump will be zero at the quasi-Fermi level separation, so the change in gain will be zero for a pump wavelength located at that energy. Finally, the change in induced device voltage due to absorption of an external optical pump light will be zero when the pump wavelength is at the transparency energy.

The transformed spontaneous emission procedure yields highly accurate measurements of the gain tail, which are difficult to obtain from the other techniques. The experimentally obtained spectra are presented along with theoretical fits based on a many-body gain model. Theoretical gain models provide estimates of the carrier density within the active region by matching the predicted gain spectrum to the measured gain. For the best accuracy it is necessary to include all of the most important physical effects which determine the gain. A non-Markovian optical gain model with many-body effects is used to calculate the spectra. The plasma screening, band-gap renormalization (BGR), and the excitonic or the Coulomb enhancement (CE) of the interband transition probability are included in the model. Good agreement between theory and experiment for both the transverse electric (TE) and transverse magnetic (TM) polarizations is obtained. Our model also provides the numerical values for the carrier densities.

Experimental results for the measurement of gain, loss, and transparency energy using the spontaneous emission spectrum and its fundamental relationship with the gain is presented in Sec. II along with a comparison to other methods for obtaining these quantities. A description of the manybody gain model and comparisons between theoretical and experimental results are discussed in Secs. III and IV. Finally, the summary and conclusions are presented in Sec. V.

\section{EXPERIMENTAL TECHNIQUES}

The following experiments were performed using a nominally lattice-matched AlGaInAs multiple quantum-well laser. An unstrained quantum-well laser is chosen so that there will be a significant amount of gain for both the TE and TM polarizations. The laser is a Fabry-Perot device with a cavity length of $638 \mu \mathrm{m}$ and one facet coated for high (85\%) reflectivity. Round trip mirror loss is $11.4 \mathrm{~cm}^{-1}$. The device was temperature controlled and all data were taken at $25^{\circ} \mathrm{C}$. For all spectra the device current was measured to within 10 $\mu \mathrm{A}$ accuracy. Threshold at this temperature was $11.7 \mathrm{~mA}$. Further structural details are described in Sec. IV.

\section{A. Method I: Measurement of spontaneous emission and the use of the fundamental relation}

As described above, direct measurements of the gain provided insufficient data in the low energy tail region. On the other hand, very accurately measured values of transparency energy and loss can be yielded by using an indirect technique based on the relationship between spontaneous emission and gain. The optical gain spectra are related to the spontaneous emission spectra from the detailed balance between absorption and emission of photons. ${ }^{5,12-15}$ That is, the modal gain from spontaneous emission is given by

$$
g(E)=\frac{h^{3} c^{2}}{8 \pi n_{r}^{2} E^{2}}\left(1-\exp \frac{E-\Delta F}{k_{B} T}\right) r_{s p}(E)
$$

in which $r_{s p}$ is the spontaneous emission rate, $\hbar=h / 2 \pi, h$ is the Planck's constant, $T$ is the temperature, $E=\hbar \omega, \Gamma$ is the optical confinement factor, and $\Delta F$ is the transparency energy, which is given by the energy separation of the quasiFermi levels of the electrons and holes. In practice, the spontaneous emission power $P_{s p}(\lambda)$ is measured within small resolution intervals $d \lambda$ determined by the spectrometer over the entire spectrum,

$$
P_{s b}(\lambda)=K_{1} \frac{h c}{\lambda} r_{s p}(\lambda) d \lambda V
$$

so the relation in Eq. (1) must be modified. First, the energy is expressed in terms of the wavelength, then the spontaneous emission rate is substituted by the power through Eq. (2). The result is 


$$
g(\lambda)=\frac{\lambda^{5}}{K_{1} 8 \pi n_{r}^{2} h c^{2} V d \lambda}\left(1-\exp \frac{\frac{h c}{\lambda}-\Delta F}{k_{B} T}\right) P_{s p}(\lambda) .
$$

Next the material gain is written in terms of the measured net modal gain as

$$
g_{m}(\lambda)=\Gamma g(\lambda)-\alpha_{i},
$$

where $\Gamma$ is the optical confinement factor and $\alpha_{i}$ is the intrinsic loss for the optical waveguide mode. Now the modal gain can be written in terms of the measured spontaneous emission power:

$$
\begin{aligned}
g_{m}(\lambda)= & \frac{\Gamma \lambda^{5}}{K_{1} 8 \pi n_{r}^{2} h c^{2} V d \lambda}\left(1-\exp \frac{\frac{h c}{\lambda}-\Delta F}{K_{B} T}\right) \\
& \times P_{s p}(\lambda)-\alpha_{i}
\end{aligned}
$$

or, more simply, as

$$
g_{m}(\lambda)=Q \lambda^{5}\left(1-\exp \frac{\frac{h c}{\lambda}-\Delta F}{k T}\right) P_{s p}(\lambda)-\alpha_{i} .
$$

In the abovce expression, there are three parameters which relate gain to spontaneous emission: the transparency energy $\Delta F$, the loss $\alpha_{i}$, and the constant $Q$, which includes the optical coupling constant. The losses, which actually vary slightly as a function of wavelength, are assumed constant in this technique. That is, the determination of the transparency energy is equivalent to determining the losses, since the material gain at that energy is zero. The coupling constant and transparency energy are therefore used as fitting parameters for each pair of gain and spontaneous emission spectra.

In principle it should be possible to fix the value of the coupling constant when matching a pair of gain and spontaneous emission spectra, and use that fixed value for subsequent matches. Alternatively, the best-fit matches of the gain and spontaneous emission spectra ought naturally to result in a uniform value for the coupling constant. Recent studies $^{20-22}$ employing this technique, however, have permitted some variation in the coupling constant from spectrum to spectrum. But allowing the coupling constant to fluctuate, even by a few percent, can lead to significant changes in the extracted values of transparency energy and loss. Therefore it is necessary to consider whether it is valid to allow the coupling constant to vary for each pair of gain and spontaneous emission spectra.

In the experiments the spontaneous emission spectra are measured from the side of the laser using a bare fiber. It is possible to mount the fiber rigidly and isolate it from vibration and air currents so that the position of the fiber is held constant. The test laser is temperature controlled and biased at a constant current, and monitored throughout the duration of the experiments. When the total spontaneously emitted power is measured and observed for stability, it varies by less than one percent over times comparable to the duration of the experiment. Thus, it does not seem reasonable to allow the coupling coefficient to vary in the fitting procedure.

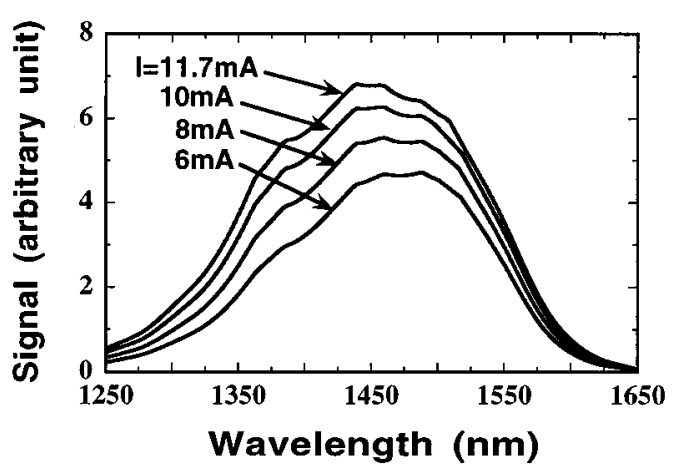

FIG. 1. The spontaneous emission spectra at four injection currents, $I=6,8$, 10 , and $11.7 \mathrm{~mA}$, in increasing order. The wavelengths corresponding to the gain spectra extending from 1500 to $1650 \mathrm{~nm}$.

It is also worthwhile to consider wavelength dependence of the coupling coefficient. A multimode silica core fiber was used to couple the spontaneous emission. Figure 1 shows the spontaneous emission for four injection currents up to laser threshold. Here the portion of the spontaneous emission spectrum which is transferred to obtain gain spectra is at wavelengths of 1500 up to $1650 \mathrm{~nm}$. In coupling to the fiber, small variations in the glass refractive index (from 1.44462 to 1.44267 ) lead to variation in the reflectivity, and a simple estimate can be made using the Fresnel formula, i.e.,

$$
R=\frac{\left(n_{e}-1\right)^{2}}{\left(n_{e}+1\right)^{2}}
$$

in which $n_{e}$ is the effective refractive index of the fundamental mode of the fiber. The reflectivity changes from $3.31 \%$ to $3.28 \%$ over the wavelength range of $1500-1650 \mathrm{~nm}$ used to match gain and spontaneous emission. Absorption within the fiber is negligible. So, a $0.1 \%$ variation in the coupling may be reasonable, though in obtaining these results the coupling constant was not varied with respect to wavelength.

Polarization dependent gain data are extracted by measuring the ASE spectra through a polarizing laser-optical fiber interface. The gain is then calculated using the HakkiPaoli method, in which the ratio of intensity maxima and minima of the Fabry-Perot modes is related to the gain. ${ }^{1}$ As mentioned earlier, this technique relies on a good signal to noise ratio, which may be insufficient for wavelengths near the band edge where the ASE intensity is extremely low. In the peak gain region for currents near threshold, the modes are almost lasing and may not be completely resolved by the spectrometer. The mode maxima become artificially lowered and the Hakki-Paoli method underestimates the gain. In this region, Cassidy's method, employing the ratio of the average mode power to the mode minimum, is less susceptible to these effects. For our gain measurements, the Hakki-Paoli technique is used at nearly all wavelengths, except near the peak gain at just below threshold, where Cassidy's method is employed.

To obtain the best fit results from Eq. (6), we choose a constant value of $Q$ to be used for each of the currents. The quasi-Fermi level of separation $\Delta F$ is then varied for each current to yield transformed spontaneous emission spectra 


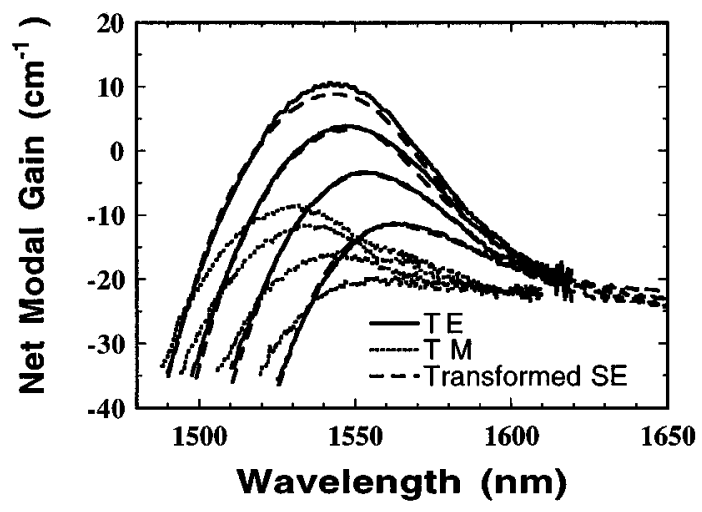

FIG. 2. Measured net modal gain spectra (solid-TE polarization, dotted-TM polarization) and transformed spontaneous emission spectra (dashed-TE polarization) are shown for injection current $I=6,8,10$, and $11.7 \mathrm{~mA}$. Note the tail regions of the transformed spectra (on the long-wavelength side), which show closer agreement for TE and TM polarizations.

with the smallest deviation from the measured modal gain. The value of $Q$ which is selected is that which gives the best simultaneous fit for all the spectra. The resulting transformed gain spectra then provide accurate gain data in the tail region where none was available from the direct measurement using the Hakki-Paoli or Cassidy's method.

The measured net modal gain spectra, $\Gamma g-\alpha_{i}$, (solid-TE polarization, dotted-TM polarization) are shown in Fig. 2 with the transformed spontaneous emission based on the above fitting techniques. Overall the agreement is quite good; but the improvement is most noticeable in the tail region, where the transformed spectra (dashed) show significantly less noise than the TE spectra (solid). It is interesting to note the tail of the TE gain spectra, which initially appear to taper off at a loss value different than that of the TM spectra. The transformed data show that the actual tail reaches a loss point in much closer agreement with the measured TM losses. The extracted values of transparency energy and loss are shown in Figs. 3(a) and 3(b), respectively. Also shown for comparison are the values obtained from the independent measurements of the loss and transparency energies $^{18}$ described below. The extracted values for all methods are in close agreement. The SE-transform method is no better or worse than the other techniques. The advantage of this method, however, is that the entire gain spectrum can be extracted from the spontaneous emission spectrum, yielding very accurately measured values of transparency energy and loss, and giving a more accurate tail for the gain spectrum.

\section{B. Method II: Crossing of polarization dependent optical gain spectra}

Figure 4 shows the polarization dependent gain spectra obtained from the facet ASE spectrum. The transparency energy may be measured directly by examining the crossing point of the TE and TM gain spectra, and the value of loss at the crossing point is equal to the intrinsic loss. The values extracted from comparison of TE and TM gain spectra are
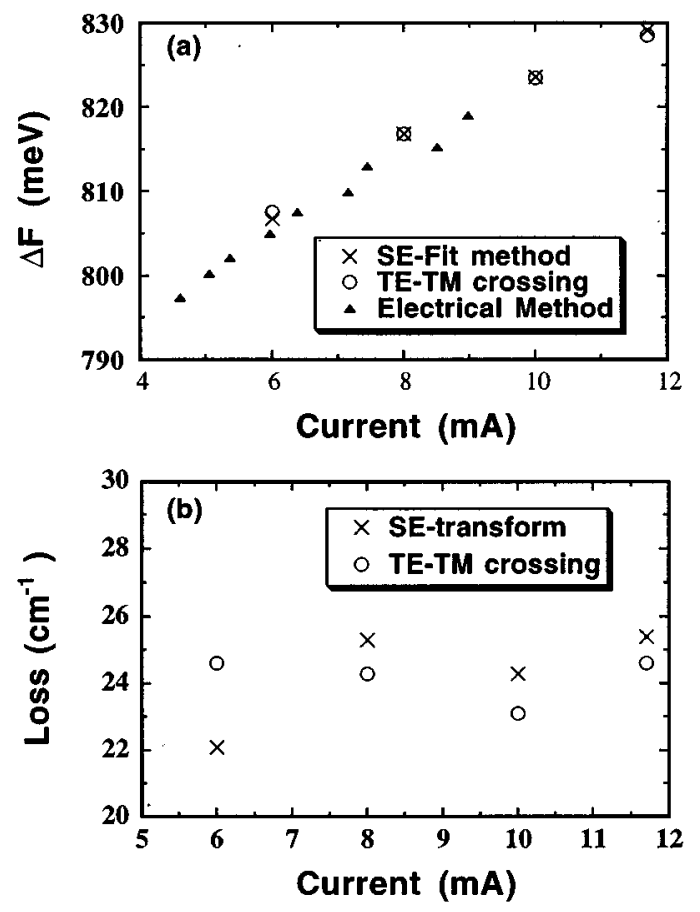

FIG. 3. A comparison of (a) the measured transparency energy based on the TE-TM crossing method (circles), the electrical method (triangles), and the spontaneous emission (SE) transformation method (crosses) and (b) the intrinsic loss using the TE-TM crossing method (circles) and the SE transformation method (crosses).

presented in Fig. 3. There are certain flaws in measuring the loss and transparency in this manner. Because of noise near the gain tail (especially for the TE spectra), it appears that the losses may be polarization dependent. If that is the case, estimates of both loss and transparency energy based on the crossing of the TE and TM gain spectra are questionable. Yet even if the losses were not polarization dependent, measuring them by examining the crossing point of TE and TM spectra is not always straightforward. For instance, the slope

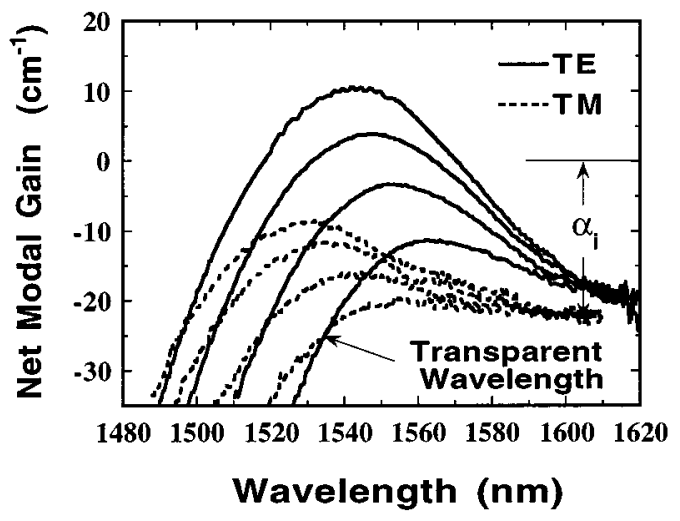

FIG. 4. Polarization dependent gain spectra for currents of $6,8,10$, and 11.7 $\mathrm{mA}$, from bottom to top curves, are shown. The plateau at the long wavelength sides gives the intrinsic loss. The crossing point of the TE- and TM-polarization gain spectra gives the transparent wavelength at each injection current. Note the apparent discrepancy in the location of the gain tails, which suggests a polarization-dependent intrinsic loss. 


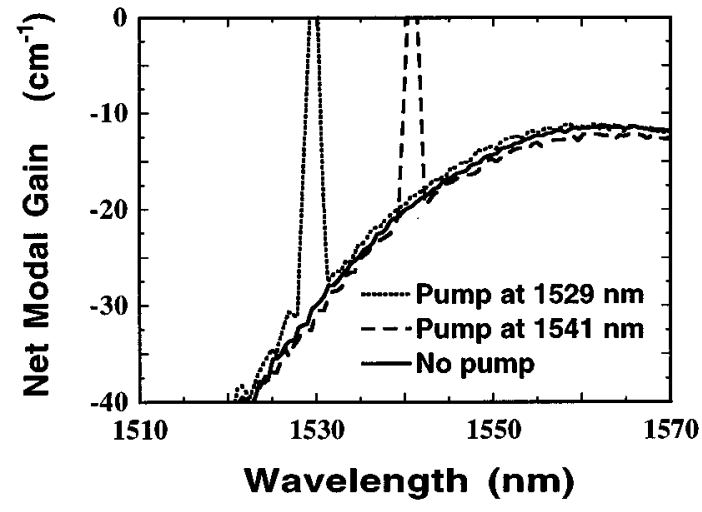

FIG. 5. Gain spectra with (dotted and dashed) and without (solid) an external optical pump. Pump light is absorbed at $1529 \mathrm{~nm}$ and amplified at 1541 $\mathrm{nm}$, causing the gain spectrum to be enhanced (dotted) or compressed (dashed), respectively.

of the gain is relatively steep near the crossing point, so that while the measured transparency energy may be relatively accurate, the exact value of loss is much less certain. A more general problem is that it is not always possible to obtain clear TM spectra, especially in compressively strained lasers. Nevertheless, measuring the TE-TM crossing is straightforward, and has the advantage of simplicity.

\section{Method III: Measurement of gain compression in the presence of an external laser pump}

One technique for measuring the transparency energy involves measuring the gain of a semiconductor laser with a constant biased current in the presence of an external optical pump. In contrast to measurements of the single-pass gain at the pump wavelength, changes in the overall gain spectrum induced by the absorption or amplification of a pump are examined. If the pump wavelength is located within the gain region, amplification of the pump will consume carriers and reduce the overall gain. Conversely, if the pump wavelength is in the absorption region, the pump will create carriers and enhance the gain. In these experiments an external distributed feedback (DFB) laser is used as the pump source, and it is filtered to prevent the ASE of the pump DFB laser from entering the Fabry-Perot laser under study. Figure 5 shows measured gain spectra at $6 \mathrm{~mA}$ with and without an external pump. First the pump is located in the absorption region at $1541 \mathrm{~nm}$, and the gain is enhanced. Then the pump is tuned to the gain region at $1529 \mathrm{~nm}$, and the gain is compressed.

The two pump wavelengths in Fig. 5 are separated by 12 $\mathrm{nm}$ or $6.3 \mathrm{meV}$, which corresponds to an uncertainty in the transparency energy of less than $1 \%$. This degree of uncertainty in the transparency energy is too large to make an accurate comparison. Fortunately, one can interpolate to improve the estimate, which gives an estimated transparency wavelength of $1535 \mathrm{~nm}$, which is in close agreement with the results obtained by TE-TM crossing method. Likewise, the estimated losses can be interpolated from the pump locations, for an intrinsic loss of $25 \mathrm{~cm}^{-1}$.

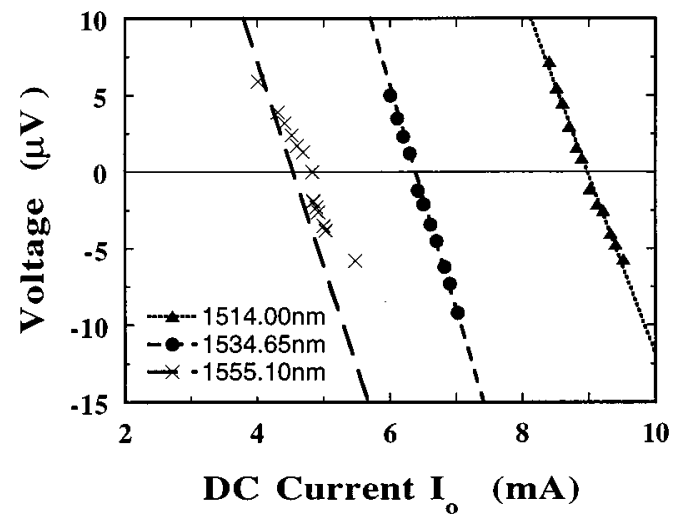

FIG. 6. A plot of the induced ac voltage magnitude across the laser terminals, due to the injection of the modulated external pump laser, as the dc bias current $\left(I_{o}\right)$ of the test laser is varied. When the magnitude of the induced ac voltage is zero, the laser wavelength is at the transparency energy.

While improvement might seem possible by pumping at wavelengths closer to transparency, in practice very high pump powers are required since the absorption or gain becomes very small. Little gain change was observed for pump wavelengths between 1529 and $1541 \mathrm{~nm}$ in experiments conducted at a bias of $6 \mathrm{~mA}$, though pump powers as high as 3 $\mathrm{mW}$ were used. Other, more subtle problems arise as well; for instance, the sensitivity of this technique varies depending on whether the pump wavelength overlaps a Fabry-Perot resonance or not. If the pump does not overlap a Fabry-Perot maximum, the gain or absorption is weaker and the gain change may be misleadingly small. A slightly different approach based on an electrical characterization technique, described in the next section, overcomes these difficulties and provides superior accuracy in measuring the transparency energy and loss.

\section{Method IV: Measurement of electrical characteristics in the presence of an external laser pump}

An excellent method ${ }^{19}$ for determining the transparency energy to a high degree of accuracy also involves the use of an external pump laser. In contrast to the preceding method, the pump signal is modulated and injected into the laser under study (test laser) while the ac signal appearing across the test laser terminals is monitored by a lock-in amplifier. A modulating frequency of a few $\mathrm{kHz}$ is sufficient for high signal to noise ratio. When using a pump filtered to remove its ASE, the magnitude of the induced ac voltage in the test laser will be a minimum when the pump laser is tuned to the transparency energy. Alternatively, the bias of the laser under test may be varied while the pump laser is fixed. The latter method was used to measure the transparency energy and loss independently. The pump sources used were several single-mode DFB laser operating between 1514 and 1555 nm. Figure 6 shows the measured ac voltage magnitude ver- 
sus current for three of the pump wavelengths used. The negative or positive sign in the plot is used to indicate the relative phase. This plot shows that as the pump wavelength is decreased, the current which has that wavelength as the transparency point increases, which is the correct trend. From our measurements, the zero crossings are obtained by fitting a curve to the measured data and taking the fitted zero value. The resulting transparency energies are compared to those of the other methods in Fig. 3(a).

\section{MANY-BODY GAIN THEORY}

The optical gain with the Lorentzian line shape function gives an anomalous absorption region below the band gap and discrepancies between the transparency points and the Fermi-level separation. The spontaneous emission transformation method using the fundamental relations between the spontaneous emission and the absorption coefficients guarantees that artifacts related to the Lorentzian line shape are circumvented independent of the line shape functions (Lorentzian, Gaussian, or others). However, the slow convergence of the Lorentzian still leads to a very long tail of the gain spectra into the band gap. ${ }^{15}$ Here, a non-Markovian (Gaussian) gain model with many-body effects under the spontaneous emission transformation method is used to calculate the optical gain, and we will show that this model agrees very well with the experimental data in Sec. IV. ${ }^{16,17}$

Under the spontaneous emission transformation method, the optical gain with non-Markovian relaxation and manybody effects is given by ${ }^{16}$

$$
\begin{aligned}
g(\omega)= & \left(1-\exp \frac{h \omega-\Delta F}{k T}\right) \sqrt{\frac{\mu_{o}}{\epsilon}}\left(\frac{e^{2}}{m_{o}^{2} \omega}\right) \\
& \times \int_{0}^{\infty} d k_{\|} \frac{k_{\|}}{\pi L_{z}}\left|M_{l m}\right|^{2} f_{1}^{c}\left(1-f_{m}^{\vee}\right)\left(1-\operatorname{Re} q_{k_{\|}}\right) \\
& \times \frac{\operatorname{Re} L\left[E_{l m}\left(k_{\|}\right)\right]-\operatorname{Im} q_{k_{\|}} \operatorname{Im} L\left[E_{l m}\left(k_{\|}\right)\right]}{\left(1-\operatorname{Re} q_{k_{\|}}\right)^{2}+\left(\operatorname{Im} q_{k_{\|}}\right)^{2}},
\end{aligned}
$$

where $\omega$ is the optical angular frequency, $\mu_{o}$ is the vacuum permeability, $\epsilon$ is the dielectric constant, $k_{\|}$is the in-plane vector, $L_{z}$ is the well thickness, $\left|M_{l m}\right|^{2}$ is the momentum matrix element in the strained $Q W, f_{l}^{c}$ and $f_{m}^{\vee}$ are the Fermi functions for the conduction band states and the valence band states, and $\Delta F$ is the difference in quasi-Fermi energy levels of the electrons and holes. The indices $l$ and $m$ denote the electron states in the conduction band and the heavy hole (light hole) subband states in the valence band. Also, $E_{l m}\left(k_{\|}\right)=E_{l}^{c}\left(k_{\|}\right)-E_{m}^{\mathrm{v}}\left(k_{\|}\right)+E_{g}+\Delta E_{S X}+\Delta E_{C H}$ is the normalized transition energy between electrons and holes, where $E_{g}$ is the band gap of the material, and $\Delta E_{S X}$ and $\Delta E_{C H}$ are the screened exchange and Coulomb-hole contributions to the band gap renormalization. The factor $q\left(k_{\|}\right)$is the excitonic or Coulomb enhancement of the interband transition probability. ${ }^{23,24}$ The line shape function $\left[E_{l m}\left(k_{\|}\right)\right]$is Gaussian for the simplest non-Markovian quantum kinetics and is given by ${ }^{16,17}$

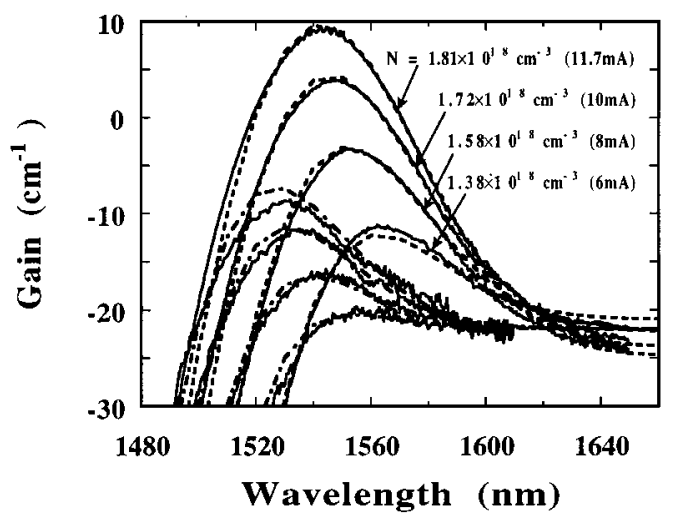

FIG. 7. The measured gain spectra (solid curves) and theoretical fits (dashed lines) using a many-body gain model are shown for both TE (upper group of curves) and TM (lower group) polarizations.

$$
\begin{aligned}
\operatorname{Re} L\left(E_{l m}\left(k_{\|}\right)\right)= & \sqrt{\frac{\pi \tau_{\text {in }}\left(k_{\|}\right) \tau_{c}}{2 \hbar^{2}}} \\
& \times \exp \left(-\frac{\tau_{\text {in }}\left(k_{\|}\right) \tau_{c}}{2 \hbar^{2}} E_{l m}^{2}\left(k_{\|}\right)\right)
\end{aligned}
$$

and

$$
\begin{aligned}
\operatorname{Im} L\left(E_{l m}\left(k_{\|}\right)\right)= & \frac{\tau_{c}}{\hbar} \int_{0}^{\infty} \exp \left(-\frac{\tau_{c}}{2 \tau_{\text {in }}\left(k_{\|}\right)} t^{2}\right) \\
& \times \sin \left(\frac{\tau_{c} E_{l m}\left(k_{\|}\right)}{\hbar} t\right) d t .
\end{aligned}
$$

The intraband relaxation time $\tau_{i n}$ and correlation time $\tau_{c}$ are assumed to be 85 and $20 \mathrm{fs}$ in our calculations, respectively, for the best fit with the experimental data. The valence band structure is calculated by using the block-diagonalized $3 \times 3$ Hamiltonian based on the $\mathbf{k} \cdot \mathbf{p}$ method. ${ }^{25}$

\section{COMPARISON OF THEORETICAL AND EXPERIMENTAL RESULTS AND DISCUSSION}

Figure 7 shows the comparison between experimental (solid curves) and calculated (dashed curves) TE and TM gain spectra for the lattice-matched InGaAlAl multi-QW laser. The structural details are summarized in Table I. Experimental data are measured at injection currents, $I=6,8,10$, and $11.7 \mathrm{~mA}$. The corresponding carrier densities are 1.38 , $1.58,1.72$, and $1.81 \times 10^{18} \mathrm{~cm}^{-3}$, respectively. The carrier

TABLE I. Structure of the laser under study.

\begin{tabular}{llllc}
\hline \hline Well: & Number of wells & 5 & SCH width: & $600 \AA$ \\
& Material & InGaAlAs & Stripe width: & $1.46 \mu \mathrm{m}$ \\
& Strain & lattice matched & Cavity length: & $638 \mu \mathrm{m}$ \\
& Width & $86 \AA$ & & \\
& PL wavelength & w.56 $\mu \mathrm{m}$ & & \\
Barrier: & Material & InGaAlAs & \\
& Strain & lattice matched & \\
& Width & $50 \AA$ & \\
& PL wavelength & $1.21 \mu \mathrm{m}$ & \\
\hline \hline
\end{tabular}



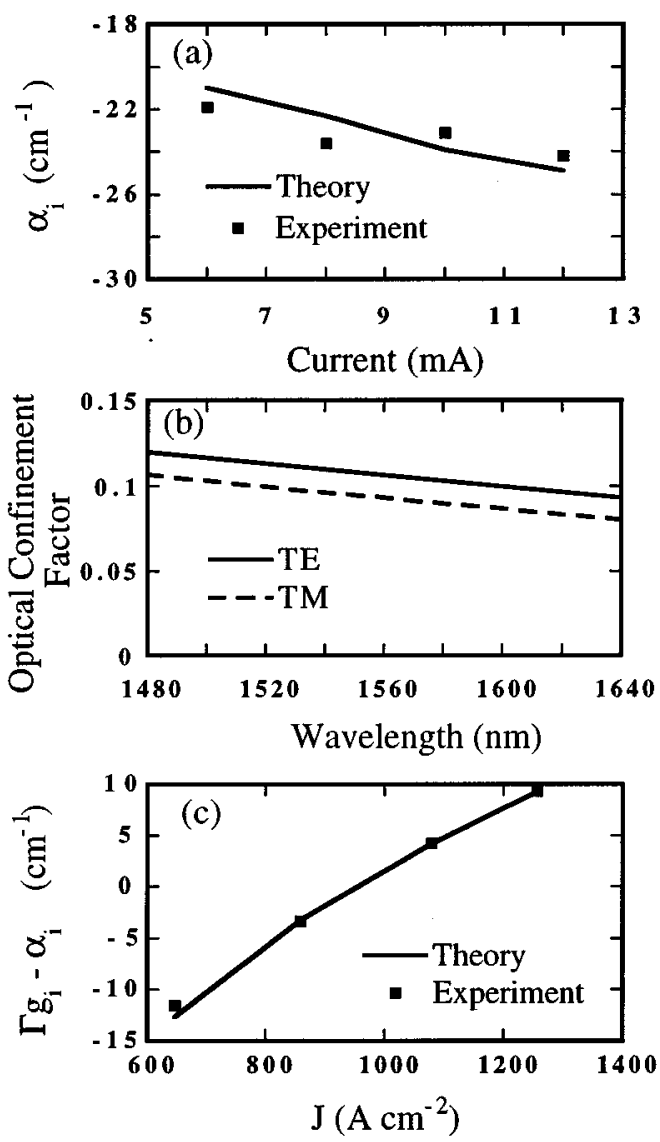

FIG. 8. The measured intrinsic loss and trend for the fits are shown in (a) for the TE polarization. In (b) the optical confinement factors for both polarizations are plotted as a function of wavelength, and in (c) the measured and predicted net modal gain versus the injection current density are shown.

densities are selected to give the best fit to the experimental data. Recently, Shtengel et al. showed that the absolute value of the optical loss increases with an increasing current. ${ }^{26}$ Here, we obtain the optical losses for the TE polarization equal to $-21,-22.3,-23.9$, and $-24.9 \mathrm{~cm}^{-1}$ for $6,8,10$, and $11.7 \mathrm{~mA}$ injection currents, respectively. These values are generally within $1 \mathrm{~cm}^{-1}$ of the best measured values and are plotted in Fig. 8(a); for the $8 \mathrm{~mA}$ spectra the losses are within $3 \mathrm{~cm}^{-1}$. The theoretical gain spectra show reasonably good agreement with the experimental results.

The optical confinement factor $\Gamma$ per unit width of the quantum well obtained from the fitting result is shown in Fig. 8 (b) and is approximately $1.8 \times 10^{-3} \mathrm{~nm}^{-1}$ at $1.5 \mu \mathrm{m}$. This value is in good agreement with that reported for InP-based quantum well lasers. ${ }^{27}$ The absolute magnitude of the TE and TM confinement factors differs by about 0.01 ; the TM polarization being less confined. Variation in the confinement factor with respect to wavelength follows the same trend for each polarization. Confinement decreases with increasing wavelength, since the ratio of the active region length or width to the wavelength is decreasing. In Fig. 8(c), the predicted and measured values of peak net modal gain versus injected current density also show excellent agreement. From these results, we know that the non-Markovian gain model with many-body effects is useful for comparison with experi- ment. If the Lorentzian line shape is used instead of the Gaussian line shape, it would be difficult to obtain the exact optical losses because the slow convergence of the Lorentzian leads to a very long tail of the gain spectra into the band gap. This is mainly due to the steepness of its low-energy tail in the Gaussian, which is similar to the line shape obtained by considering the carrier-carrier and carrier-longitudinal optical (LO) phonon scatterings. ${ }^{7,8}$

\section{SUMMARY AND CONCLUSIONS}

High accuracy measurements of gain, loss and transparency energy in long-wavelength semiconductors based on a hybrid approach using the fundamental relationship between the gain and the spontaneous emission spectra have been demonstrated. Our hybrid approach for the gain spectrum alleviates many of the problems related to the poor signal to noise ratio in the ASE near and below the band edge, yielding more accurate gain data in this region than any of the other available techniques. The values of loss and transparency energy obtained from the spontaneous emission transformation method agree favorably with those obtained from other techniques such as the measurement of polarization dependent gain, and measurements of the gain compression and electrical characteristics in the presence of an optical pump beam. These independent measurements of transparency energy and loss provide validation that our correlated gain and spontaneous emission spectra have a high degree of reliability. We have also compared the measured gain spectra for both polarizations with calculations using a nonMarkovian gain model with many-body effects under the spontaneous emission transformation method. This model yields very good agreement to the measured gain spectra.

\section{ACKNOWLEDGMENTS}

This work was support by ONR under Grant Nos. N00014-96-10303 and AASERT N00014-96-1-0902. S. H. Park was also partially supported by Catholic University of Taegu-Hyosang.

${ }^{1}$ B. W. Hakki and T. L. Paoli, J. Appl. Phys. 46, 1299 (1975).

${ }^{2}$ D. T. Cassidy, J. Appl. Phys. 56, 3096 (1984).

${ }^{3}$ V. Jordan, IEE Proc.: Optoelectron. 141, 13 (1994).

${ }^{4}$ M. Yamada and Y. Suematsu, J. Appl. Phys. 52, 2653 (1981).

${ }^{5}$ P. Rees and P. Blood, IEEE J. Quantum Electron. 31, 1047 (1995).

${ }^{6}$ M. Yamanishi and Y. Lee, IEEE J. Quantum Electron. 23, 367 (1987).

${ }^{7}$ M. Asada, Quantum Well Lasers, edited by P. S., Zory, Jr. (Academic, San Diego, CA, 1993), Chap. 2.

${ }^{8}$ W. W. Chow, P. M. Smowton, P. Blood, A. Girndt, F. Jahnke, and S. W. Koch, Appl. Phys. Lett. 71, 157 (1997).

${ }^{9}$ S. H. Park and S. L. Chuang, Appl. Phys. Lett. 72, 3103 (1998)

${ }^{10}$ C. F. Hsu, P. S. Zory, C. H. Wu, and M. A. Emanuel, IEEE J. Sel. Top. Quantum Electron. 3, 158 (1997).

${ }^{11}$ S. H. Park and S. L. Chuang, Appl. Phys. Lett. 72, 287 (1998).

${ }^{12}$ G. Lasher and F. Stern, Phys. Rev. A 133, 553 (1964).

${ }^{13}$ C. H. Henry, R. A. Logan, and F. R. Merritt, J. Appl. Phys. 51, 3042 (1980).

${ }^{14}$ S. L. Chuang, J. O'Gorman, and A. H. J. Levi, IEEE J. Quantum Electron. 29, 1631 (1993).

${ }^{15}$ P. M. Enders, IEEE J. Quantum Electron. 33, 580 (1997).

${ }^{16}$ D. Ahn, Prog. Quantum Electron. 21, 249 (1997).

${ }^{17}$ S. H. Park and S. L. Chuang, Proc. SPIE 3283, 314 (1998).

${ }^{18}$ G. E. Shtengel and D. A. Ackermann, Electron. Lett. 31, 1157 (1995). 
${ }^{19}$ P. A. Andrekson, N. A. Olsson, T. Tanbun-Ek, R. A. Logan, D. L. Coblentz, and H. Temkin, Electron. Lett. 28, 171 (1992).

${ }^{20}$ G. Hunziker, W. Knop, P. Unger, and C. Harder, IEEE J. Quantum Electron. 31, 643 (1995)

${ }^{21}$ A. Schoenfelder, J. D. Ralston, K. Czotscher, S. Weisser, J. Rosenzweig, and E. C. Larkins, J. Appl. Phys. 80, 582 (1996).

${ }^{22}$ W. Fang, M. Hattendorf, S. L. Chuang, J. Minch, C. S. Chang, C. G. Bethea, and Y. K. Chen, Appl. Phys. Lett. 70, 796 (1997).

${ }^{23}$ H. Haug and S. W. Koch, Quantum Theory of the Optical and Electronic
Properties of Semiconductors (World Scientific, Singapore, 1993).

${ }^{24}$ W. W. Chow, S. W. Koch, and M. Sergent III, Semiconductor-Laser Physics (Springer, Berlin, 1993).

${ }^{25}$ C. S. Chang and S. L. Chuang, IEEE J. Sel. Top. Quantum Electron. 1, 218 (1995).

${ }^{26}$ G. E. Shtengel, P. A. Morton, R. F. Kazarinov, D. A. Ackerman, M. S. Hybertsen, G. L. Belenky, and C. L. Reynolds Jr., Proc. SPIE 2994, 678 (1997).

${ }^{27}$ J. P. Loehr and J. Singh, IEEE J. Quantum Electron. 27, 708 (1991). 